Journal of Patient-Centered

Volume 2

Issue 4 -- Integrative Medicine

Article 15

$11-20-2015$

\title{
Geographic Distribution of Infant Death During Birth Hospitalization and Maternal Group B Streptococcus Colonization: Eastern Wisconsin
}

Jessica J.F. Kram

Dennis J. Baumgardner

Kiley A. Bernhard

Melissa A. Lemke

Follow this and additional works at: https://aah.org/jpcrr

Part of the Bacteria Commons, Congenital, Hereditary, and Neonatal Diseases and Abnormalities Commons, and the Women's Health Commons

\section{Recommended Citation}

Kram JJ, Baumgardner DJ, Bernhard KA, Lemke MA. Geographic distribution of infant death during birth hospitalization and maternal Group B Streptococcus colonization: eastern Wisconsin. J Patient Cent Res Rev. 2015;2:205-206. doi: 10.17294/2330-0698.1225

Published quarterly by Midwest-based health system Advocate Aurora Health and indexed in PubMed Central, the Journal of Patient-Centered Research and Reviews (JPCRR) is an open access, peer-reviewed medical journal focused on disseminating scholarly works devoted to improving patient-centered care practices, health outcomes, and the patient experience. 
included nongender-based $(<15 \mathrm{ml} /$ beat $)$, gender-based ( $<15 \mathrm{ml} /$ beat for males and $<10 \mathrm{ml} /$ beat for females) and $<80 \%$ of $\mathrm{O}_{2}$ pulse based on five different definitions of predicted $\mathrm{VO}_{2}$ max. The optimal cutoff obtained was then used to create the composite criterion. For the purpose of evaluating this composite criterion, the study population was recategorized as: noncardiac group $(n=18)$, normal patients according to the composite criterion; or cardiac group $(n=13)$, abnormal patients according to the composite criterion. Patients who were normal by only one component of the composite criterion were categorized as borderline $(n=23)$. Data were analyzed against the comprehensive CPX test by first excluding the borderline patients and then by including them with either the cardiac or noncardiac group.

Results: The 6-variable algorithm performed well against comprehensive CPX test in discriminating cardiac from noncardiac causes of dyspnea, with 94\% sensitivity, 92\% specificity, $84 \%$ positive predictive value (PPV), 97\% negative predictive value (NPV) and 93\% accuracy. The results remained consistent for gender and referral source. $\mathrm{O}_{2}$ pulse, as defined by Wasserman, had the highest accuracy, specificity and PPV and therefore was used to define the composite criterion. The composite criterion had an accuracy of $87 \%$, PPV of $77 \%$, NPV of $94 \%$, sensitivity of $91 \%$ and specificity of $85 \%$, when borderline patients were excluded. Including borderline patients in the cardiac group $(n=36)$ improved sensitivity $(94 \%)$ and maintained NPV (94\%) but greatly decreased specificity (46\%), PPV (44\%) and accuracy (61\%), whereas including these patients in the noncardiac group $(n=41)$ improved specificity (92\%) and maintained similar PPV (77\%) and accuracy (81\%) but decreased sensitivity (59\%) and NPV (83\%).

Conclusion: This is the first study to validate a diagnostic algorithm for patients undergoing CPX testing as well as demonstrate that a simplified 6-variable algorithm applied by a cardiologist without prior CPX experience is quite accurate to evaluate the optimal $\mathrm{O}_{2}$ pulse value at peak stress for discrimination of cardiac and noncardiac causes, and to provide the operating test characteristics for the common clinical practice of using composite criterion to diagnose cardiac versus noncardiac causes of dyspnea.

\section{RIESELBACH DISTINGUISHED PAPERS \#2-4}

See page 217 for citations.

\section{SELECT ABSTRACTS \\ Score Big for Decreasing Mortality: ICD Risk Score Model}

\author{
Linda Francaviglia, Rachel Petersen, Maria Stone, M. \\ Eyman Mortada
}

Departments of Cardiovascular Data Services and Quality Management, Aurora Cardiovascular Services, Aurora Health Care

Background: Aurora Health Care, a system of 14 acute care hospitals in eastern Wisconsin, has been a long-time participant in the American College of Cardiology's National Cardiovascular Data Registries, submitting data to its ICD Registry $^{\mathrm{TM}}$ since 2005. Our system's implantable cardioverterdefibrillator (ICD) procedure volume averages 930 cases annually. During 2012 we experienced an increase in in-hospital mortality/morbidity for ICD cases.

Purpose: A single-center study examining in-hospital mortality/morbidity post-ICD implant before and after changes in practice and patient selection.

Methods: ICD implants and generator changes discharged from January 1, 2009, to December 31, 2012, were included in developing a risk model predicting in-hospital mortality/ morbidity. The risk score was shared with physicians for clinical input. A point system was developed, including those factors with highest risk. Using the defined factors, a risk score $>14$ was used to indicate those at highest risk for morbidity/mortality. The risk score model was fit on the development group (2009-2012), and then re-run for the intervention cohort from January 1, 2013, to June 30, 2014. Logistic regression was used in the risk model development and validation. Continuous variables were compared using Student's t-test, and categorical variables were compared using chi-square test.

Results: From 2009 to 2012, 3,417 ICD implants and generator changes were performed and included in risk model development. Of those, $200(5.9 \%)$ patients were indicated as high risk with a score $>14$. From January 2013 to June 2014, 1,057 implants and generator changes were performed, with $41(3.4 \%)$ patients indicated as high risk with a score $>14$. In the development phase, mean age was 67 years and $70 \%$ of patients were male. Post-model development, mean age was 66 years with $72 \%$ male. For patients indicated as high risk, in-hospital mortality/morbidity dropped from $20(10 \%)$ to $2(4.9 \%)$, though the decrease was not statistically significant $(\mathrm{P}=0.39)$.

Conclusion: Awareness of high-risk patients and changes in patient selection can lead to improvement in in-hospital mortality/morbidity among those high-risk patients. Although the improvement was not statistically significant, this was most likely due to low volumes and we will continue to monitor outcomes among these patients.

\section{Geographic Distribution of Infant Death During Birth Hospitalization and Maternal Group B Streptococcus Colonization: Eastern Wisconsin}

Jessica J.F. Kram, Dennis J. Baumgardner, Kiley A. Bernhard, Melissa A. Lemke

Center for Urban Population Health; Department of Family Medicine, Aurora UW Medical Group; TRIUMPH, University of Wisconsin-Madison

Background: Neonatal death rate in the United States is 4/1,000 live births; infant death rate is $6 / 1,000$. Group B Streptococcus (GBS) may be transmitted from a colonized mother (rates vary from $15 \%$ to $35 \%$ ) to the newborn during a vaginal delivery, and may contribute to neonatal death. 
Purpose: To explore the geographic distribution and associated risk factors for maternal GBS colonization and infant death prior to discharge in eastern Wisconsin births.

Methods: Retrospective study of institutional data from PeriData.net, a comprehensive birth registry, utilizing data from 2007 through 2013 at all Aurora medical centers. Categorical variables were analyzed with chi-square tests, and ordinal or continuous variables by Mann-Whitney or two-sample t-tests. Binary regression was used for multivariate modeling.

Results: Population demographics $(\mathrm{N}=99,305)$ were mean age 28 years, 59\% married, 64\% white, $42 \%$ governmentinsured, 39\% nulliparous, mean prepregnancy body mass index (BMI) of 27, gestational age of 39 weeks, birth weight of 3,296 $\mathrm{g}$ and $26 \% \mathrm{C}$-section rate. The GBS colonization rate was 22.3\%. Among ZIP codes with > 100 subjects, 8 ZIP codes had a GBS-positive rate $>30 \%$ (7 in Milwaukee, 1 in Kohler). GBS colonization was higher in blacks (34\%) than whites $(20 \% ; \mathrm{P}<0.0001)$, in unmarried women $(26 \%$ vs. $20 \%$; $\mathrm{P}<0.0001$ ), with increasing BMI (mean BMI 27.3 if GBS-positive vs. 26.6; $\mathrm{P}<0.0001$ ) and based on ZIP code group ( $\mathrm{P}<0.0001)$; and was predictive of neonatal antibiotics for sepsis $(26 \%$ if GBS-positive vs. $22 \%$; $<<0.0001)$. In multivariate analysis, unmarried status, higher BMI, race and ZIP code were predictive of GBS colonization. Rate of infant death during birth hospitalization was $0.57 \%(n=558)$ and varied by ZIP code group. GBS colonization was negatively associated with infant death $(0.25 \%$ in GBS-positive vs. $0.66 \% ; \mathrm{P}<0.0001 ; \mathrm{N}=98,065$ with lethal anomalies and stillbirths excluded). This association remained when controlling for gestational age. In multivariate analysis, death rate was associated with one ZIP code group, no prenatal care, preterm labor, vaginal bleeding, hydramnios, oligohydramnios, lower gestational age and maternal GBS (negative predictor).

Conclusion: Geographic characteristics are associated with infant death during birth hospitalization and maternal GBS colonization. Demographic characteristics are only associated with maternal GBS colonization. It is unclear if maternal GBS colonization is "protective" against infant demise due to increased surveillance.

\section{An Automated Model Using Electronic Health Record Data to Identify Delirium Among Hospitalized Older Adults: A Pilot Project}

Ariba Khan, Maharaj Singh, Hina Singh, Ayesha Maria, Michelle Simpson

Department of Geriatrics, Aurora UW Medical Group and Aurora Sinai Medical Center; Aurora Research Institute, Aurora Health Care

Background: Delirium is a serious change in mental status with adverse outcomes, but remains underrecognized. The electronic health record (EHR) may assist in the identification of delirium.

Purpose: This study was performed to generate an automated delirium identification model using data from the EHR among hospitalized older adults.

Methods: Inpatients 65 years and older were included in this cross-sectional study. The researchers used "confusion assessment method" as the gold standard to identify delirium. Four categories of variables were obtained from the EHR on the day of and the day prior to researcher assessment: 1) hypoactive delirium (any one of the following: nurse's assessment of motor retardation or reduced level of consciousness or decline in activities of daily living [ADL] score); 2) hyperactive delirium (any one of the following: use of restraints or antipsychotic medications or nurse's assessment noting a change in mental status or poor attention or motor agitation or poor thought process or anxiety); 3) patient factors (any one of the following: dementia, age, mean blood urea nitrogen and serum creatinine); and 4) health care-associated factors (any one of the following: urinary catheter, surgical procedure, brain imaging). Relationships were analyzed using chi-square or Fisher's test as appropriate. Statistical significance was set at $\mathrm{P}<0.05$. Results: Ninety-two participants in three hospitals were included in the analysis. Of these, mean age was $77 \pm 8.8$ years and $54 \%$ were female, $70 \%$ had a Morse fall score $>45$, and mean ADL score was 10 of 12 . The prevalence of delirium was $17 \%$. In the univariate analysis, variables associated with delirium included abnormal mental status ( $94 \%$ vs. $41 \%$; $\mathrm{P}<0.0001$ ); reduced level of consciousness $(69 \%$ vs. $9 \% ; \mathrm{P}<0.0001)$, motor retardation $(50 \%$ vs. $13 \%$; $\mathrm{P}<0.0007)$, motor agitation ( $38 \%$ vs. $7 \% ; \mathrm{P}=0.004)$ abnormal attention $(81 \%$ vs. $12 \% ; \mathrm{P}<0.0001)$, abnormal thought process (56\% vs. $11 \%$; $\mathrm{P}<0.001)$, dementia $(31 \%$ vs. $11 \% ; \mathrm{P}=0.03$ ), age ( 82 vs. 72 years; $\mathrm{P}=0.02$ ), number of medications ( 10 vs. $12 ; \mathrm{P}=0.0313$ ), use of antipsychotic medication ( $31 \%$ vs. $7 \%$; $\mathrm{P}=0.004)$, mean Braden score ( 15 vs. $18 ; \mathrm{P}=0.0038)$ and Morse fall score $>45(94 \%$ vs. $59 \% ; \mathrm{P}=0.02)$. In the multivariate analysis, factors associated with delirium included reduced level of consciousness and abnormal attention (area under curve 0.920).

Conclusion: This pilot study demonstrates that variables present in the EHR may be used to develop an automated model to identify delirium in hospitalized older adults. These findings need to be validated in a larger study and define if the model performs well in predicting clinical outcomes.

\section{Prognostic Indices for Hospitalized Older Adults: A Meta-Analysis and Systematic Review}

Ariba Khan, Ayesha Maria, James Hocker, Maharaj Singh, Michelle Simpson

Department of Geriatrics, Aurora UW Medical Group and Aurora Sinai Medical Center; Aurora Research Institute, Aurora Health Care

Background: A prognostication predictive model incorporated into the electronic health record (EHR) may be useful in assisting the health care team in accurately predicting mortality and may be used in appropriately 